\title{
On Ground States of Discrete $p(k)$-Laplacian Systems in Generalized Orlicz Sequence Spaces
}

\author{
Juhong Kuang ${ }^{1,2}$ \\ ${ }^{1}$ School of Mathematics and Computational Sciences, Wuyi University, Jiangmen 529020, China \\ ${ }^{2}$ Key Laboratory of Mathematics and Interdisciplinary Sciences of Guangdong, Higher Education Institutes, \\ Guangzhou University, Guangzhou 510006, China
}

Correspondence should be addressed to Juhong Kuang; kuangjuhong1982@21cn.com

Received 29 January 2014; Accepted 25 March 2014; Published 13 April 2014

Academic Editor: Yuming Chen

Copyright (C) 2014 Juhong Kuang. This is an open access article distributed under the Creative Commons Attribution License, which permits unrestricted use, distribution, and reproduction in any medium, provided the original work is properly cited.

Using the critical point theory, we establish sufficient conditions on the existence of ground states for discrete $p(k)$-Laplacian systems. Our results considerably generalize some existing ones.

\section{Introduction and Main Results}

The aim of this paper is to study the existence of ground state for discrete $p(k)$-Laplacian system

$$
\begin{aligned}
& \Delta\left[a(k)|\Delta u(k-1)|^{p(k)-2} \Delta u(k-1)\right] \\
& \quad-q(k)|u(k)|^{p(k)-2} u(k)+f(k, u(k))=0,
\end{aligned}
$$

where $p(k)>1$, for all $k \in Z, a(k)$ and $q(k)$ are real valued on $Z$. $f: Z \times R \rightarrow R$ is continuous in the second variable. Moreover, $\Delta$ is the forward difference operator defined by $\Delta u(k)=u(k+1)-u(k)$.

We may think of (1) as being a discrete analogue of the following differential system:

$$
\begin{aligned}
& \frac{d}{d t}\left(a(t)|\dot{u}(t)|^{p(t)-2} \dot{u}(t)\right) \\
& \quad-q(t)|u(t)|^{p(t)-2} u(t)+f(t, u(t))=0 .
\end{aligned}
$$

For the case $p(t) \equiv p$, system (2) is a $p$-Laplacian system, which has been widely studied; to mention a few, see $[1,2]$. Even for the special case $p=2$, system (2) can be regarded as the more general form of Emden-Fowler equation appearing in the study of astrophysics, fluid mechanics, gas dynamics, nuclear physics, relativistic mechanics, and chemically reacting system in terms of various special forms of $f(t, u(t))$ (see, e.g., [3]). The more general differential operator (2), namely, the so-called $p(t)$-Laplacian, has been studied by Fan et al. [4-7]. The $p(t)$-Laplacian operator can be used to describe the physical phenomena with "pointwise different properties." The $p(t)$-Laplacian operator has more complicated properties than that of the $p$-Laplacian; for example, it is not homogeneous, and this makes some classic theories and methods, such as the theory of Sobolev spaces, not applicable.

With the theory of nonlinear discrete dynamical systems being widely used to study discrete models appearing in many fields such as economics, ecology, computer science, neural networks, and cybernetics [8], the existence of solutions of discrete dynamical systems has become a hot topic; to mention a few, see [9-15]. For the case $p(k) \equiv p$, Iannizzotto and Tersian [16] obtained multiple homoclinic solutions for system (1) by using the critical point theorem, and for the special case $p=2, \mathrm{Ma}$ and Guo $[13,14]$ provided some sufficient conditions on the existence of homoclinic solutions for system (1). For the more general case- $p(k)$ Laplacian system (1)-Chen et al. [17] established some existence criteria to guarantee that the system has at least one or infinitely many homoclinic orbits. Motivated by Liu [2], which discussed the existence of ground state for $p$-Laplacian system, in this paper, we will consider the existence of ground state for the $p(k)$-Laplacian system (1).

Now we are in a position to state our main results. 
Theorem 1. Assume the following conditions hold:

(a) $a(k)>0$ for all $k \in Z$,

(q) $q(k)>0$ for all $k \in Z$ and $q(k) \rightarrow+\infty$ as $|k| \rightarrow+\infty$,

(p) $1<p^{-}=\inf _{k \in Z} p(k) \leq \sup _{k \in Z} p(k)=p^{+}<+\infty$ for all $k \in Z$,

(f) $f(k, u)=f_{1}(k, u)-f_{2}(k, u)$ is continuous in $u$ for all $k \in Z$, and $F(k, u)=\int_{0}^{u} f(k, s) d s$ for $u \in R$. Moreover,

$\frac{|f(k, u)|}{q(k)|u|^{p(k)-1}} \longrightarrow 0$ as $u \longrightarrow 0$ uniformly in $k \in Z$;

$\left(\mathrm{f}_{1}\right)$ there exists a constant $\beta>p^{+}$such that

$u f_{1}(k, u) \geq \beta F_{1}(k, u)>0 \quad \forall k \in Z, u \in R \backslash\{0\}$,

where $F_{1}(k, u)=\int_{0}^{u} f_{1}(k, s) d s$ for $u \in R$;

$\left(\mathrm{f}_{2}\right)$ there exists a constant $\tau \in(0, \beta)$ such that

$$
\begin{array}{r}
F_{2}(k, u) \geq 0, \quad u f_{2}(k, u) \leq \tau F_{2}(k, u) \\
\forall k \in Z, \quad u \in R,
\end{array}
$$

where $F_{2}(k, u)=\int_{0}^{u} f_{2}(k, s) d s$ for $u \in R$.

Then (1) has a ground state solution.

Remark 2. (q) implies that there exists $q_{*}>0$ such that $q(k) \geq$ $q_{*}$ for all $k \in Z$.

Remark 3. We extend Theorem 3.1 in [14] to the more general case- $p(k)$-Laplacian system. Furthermore, we obtain the existence of the ground state.

The rest of this paper is organized as follows. In Section 2, we establish the variational structure associated with (1). Some preliminary results are also provided in this section. In Section 3, we give the proof of the main result.

\section{Variational Structure and Some Preliminary Results}

In this section, we establish a variational structure which enables us to reduce the existence of solutions for (1) to the existence of critical points of the corresponding functional.

Let $S$ be the set of all two-sided sequences; that is,

$$
S=\{u=\{u(k)\}: u(k) \in R, k \in Z\} .
$$

Then $S$ is a vector space with $a u+b v=\{a u(k)+b v(k)\}$ for $u, v \in S, a, b \in R$.

We define $l^{p(k)}$ as the set of all functions $u \in S$ such that

$$
l^{p(k)}=\left\{u \in S: \sum_{k \in Z}|u(k)|^{p(k)}<+\infty\right\}
$$

with the norm

$$
\|u\|_{p(k)}=\inf \left\{r>0: \sum_{k \in Z}\left|\frac{u(k)}{r}\right|^{p(k)} \leq 1\right\} .
$$

We also define

$$
\begin{aligned}
E=\left\{u \in S: \sum_{k \in Z}\right. & {\left[a(k)|\Delta u(k-1)|^{p(k)}\right.} \\
& \left.\left.+q(k)|u(k)|^{p(k)}\right]<+\infty\right\}
\end{aligned}
$$

with the norm

$$
\begin{aligned}
\|u\|=\inf \left\{r>0: \sum_{k \in Z}\right. & {\left[a(k)\left|\frac{\Delta u(k-1)}{r}\right|^{p(k)}\right.} \\
& \left.\left.+q(k)\left|\frac{u(k)}{r}\right|^{p(k)}\right] \leq 1\right\} .
\end{aligned}
$$

We call the space $E$ a sequence space; it is a special kind of generalized Orlicz sequence space. For the general theory of generalized Orlicz spaces, see $[18,19]$.

Consider the functional $I$ on $E$ defined by

$$
\begin{gathered}
I(u)=\sum_{k \in Z}\left[\frac{a(k)}{p(k)}|\Delta u(k-1)|^{p(k)}+\frac{q(k)}{p(k)}|u(k)|^{p(k)}\right. \\
-F(k, u(k))] .
\end{gathered}
$$

Using the similar arguments as [17], we have the following lemmas.

Lemma 4. $\left(l^{p(k)},\|\cdot\|_{p(k)}\right)$ is a reflexive Banach space. Let $u \in$ $l^{p(k)}$ and

$$
\phi(u)=\sum_{k \in Z}|u(k)|^{p(k)}
$$

one has

$$
\begin{aligned}
& \text { (1) if }\|u\|_{p(k)}>1 \text {, then }\|u\|_{p(k)}^{p^{-}} \leq \phi(u) \leq\|u\|_{p(k)}^{p^{+}} \text {; } \\
& \text { (2) if }\|u\|_{p(k)}<1 \text {, then }\|u\|_{p(k)}^{p^{+}} \leq \phi(u) \leq\|u\|_{p(k)}^{p^{-}} \text {. }
\end{aligned}
$$

Lemma 5. $(E,\|\cdot\|)$ is a reflexive Banach space. Let $u \in E$ and

$$
\varphi(u)=\sum_{k \in Z}\left[a(k)|\Delta u(k-1)|^{p(k)}+q(k)|u(k)|^{p(k)}\right]
$$

one has
(1) if $\|u\|>1$, then $\|u\|^{p^{-}} \leq \varphi(u) \leq\|u\|^{p^{+}}$;
(2) if $\|u\|<1$, then $\|u\|^{p^{+}} \leq \varphi(u) \leq\|u\|^{p^{-}}$. 
Lemma 6. $I \in C^{1}(E, R)$ and the Fréchet derivative is given by

$$
\begin{aligned}
\left\langle I^{\prime}(u), v\right\rangle=\sum_{k \in Z}\left[a(k)|\Delta u(k-1)|^{p(k)-2}\right. & \\
& \times \Delta u(k-1) \Delta v(k-1) \\
& \left.+q(k)|u(k)|^{p(k)-2} u(k) v(k)\right] \\
& -\sum_{k \in Z} f(k, u(k)) v(k),
\end{aligned}
$$

for all $u, v \in E$. Moreover, the nonzero critical points of the functional I on $E$ are the nontrivial solutions of (1).

Lemma 7. $\left(f_{1}\right)$ and $\left(f_{2}\right)$ imply that

$$
u f(k, u) \geq \beta F(k, u) \quad \forall k \in Z, u \in R
$$

Moreover, for every $k \in Z$ and $u \in R, s^{-\beta} F_{1}(k, s u)$ is nondecreasing on $(0,+\infty)$ and $s^{-\tau} F_{2}(k, s u)$ is nonincreasing on $(0,+\infty)$.

Let

$$
c_{\min }=\inf \left\{I(u): I^{\prime}(u)=0, u \in E \backslash\{0\}\right\} .
$$

Then $u_{0} \neq 0$ with $I\left(u_{0}\right)=c_{\text {min }}$ is said to be a ground state solution of (1).

As usual, we make use of the following basic notations. Let $H$ be a Hilbert space and $C^{1}(H, R)$ denote the set of functionals that are Fréchet differentiable and their Fréchet derivatives are continuous on $H$.

Definition 8. Let $I \in C^{1}(H, R)$. A sequence $\left\{x_{j}\right\} \subset H$ is called a Palais-Smale sequence (P.S. sequence) for $I$ if $\left\{I\left(x_{j}\right)\right\}$ is bounded and $I^{\prime}\left(x_{j}\right) \rightarrow 0$ as $j \rightarrow+\infty$. We say that $I$ satisfies the Palais-Smale condition (P.S. condition) if any P.S. sequence for $I$ possesses a convergent subsequence.

Let $B_{r}$ be the open ball in $H$ with radius $r$ and center 0 , and let $\partial B_{r}$ denote its boundary.

Lemma 9 (mountain pass lemma). Let $H$ be a real Hilbert space and $I \in C^{1}(H, R)$ satisfies the P.S. condition. Assume that $I(0) \leq 0$ and the following two conditions hold.

$\left(\mathrm{J}_{1}\right)$ There exist constants $a>0$ and $\rho>0$ such that $I_{\partial B_{\rho}} \geq$ a.

$\left(\mathrm{J}_{2}\right)$ There exists an $e \in H \backslash B_{\rho}$ such that $I(e) \leq 0$.

Then I possesses a critical value $c \geq a$. Moreover, $c$ can be characterized as

$$
c=\inf _{h \in \Gamma} \max _{s \in[0,1]} I(h(s)),
$$

where

$$
\Gamma=\{h \in C([0,1], H): h(0)=0, h(1)=e\} .
$$

\section{Proof of Main Result}

In order to prove Theorem 1, we first prove the following lemmas.

Lemma 10. The embedding $E \hookrightarrow l^{p(k)}$ is compact.

Proof. Let $\left\{u_{j}\right\}$ be a bounded sequence in $E$; that is, there exists $M>0$ such that $\left\|u_{j}\right\|<M$ for all $j \in Z^{+}$. By reflexivity, passing to a subsequence we have $u_{j} \rightarrow u$ in $E$ for some $u \in E$. We may assume $u=0$, in particular $u_{j}(k) \rightarrow 0$ as $j \rightarrow+\infty$ for all $k \in Z$. For all $\epsilon>0$, we can find $h \in Z^{+}$such that

$$
q(k)>\frac{1+M}{\epsilon} \quad \forall|k|>h .
$$

By continuity of the finite sum, there exists $\nu_{0} \in Z^{+}$such that

$$
\sum_{|k| \leq h}\left|u_{j}(k)\right|^{p(k)} \leq \frac{\epsilon}{1+M} \quad \forall j>v_{0} .
$$

So for all $j \geq v_{0}$ we have

$$
\begin{aligned}
\sum_{k \in Z}\left|u_{j}(k)\right|^{p(k)} & \leq \frac{\epsilon}{1+M}+\frac{\epsilon}{1+M} \sum_{|k|>h} q(k)\left|u_{j}(k)\right|^{p(k)} \\
& \leq \frac{\epsilon}{1+M}+\frac{\epsilon}{1+M} \sum_{k \in Z} q(k)\left|u_{j}(k)\right|^{p(k)} .
\end{aligned}
$$

Since

$$
\sum_{k \in Z} q(k)\left|u_{j}(k)\right|^{p(k)} \leq \varphi\left(u_{j}\right) \leq \begin{cases}\left\|u_{j}\right\|^{p-}, & \text { if }\left\|u_{j}\right\|<1, \\ 1, & \text { if }\left\|u_{j}\right\|=1, \\ \left\|u_{j}\right\|^{p+}, & \text { if }\left\|u_{j}\right\|>1,\end{cases}
$$

letting $M_{0}=\max \left\{M^{p^{-}}, 1, M^{p+}\right\}$, we have

$$
\sum_{k \in Z}\left|u_{j}(k)\right|^{p(k)} \leq \frac{1+M_{0}}{1+M} \epsilon \quad \forall j \geq v_{0} .
$$

Thus, $u_{j}(k) \rightarrow 0$ in $l^{p^{(k)}}$, and the proof is completed.

Lemma 11. Assume that $u \in l^{p(k)}$ and $v \in l^{r(k)}$. Moreover, $p(k)$ satisfies condition $(p)$ and $1 / r(k)+1 / p(k)=1$ for all $k \in Z$. Then

$$
\sum_{k \in Z} u(k) v(k) \leq\left(\frac{1}{p^{-}}+\frac{1}{r^{-}}\right)\|u\|_{p(k)}\|v\|_{r(k)},
$$

where $r^{-}=\inf \{r(k): k \in Z\}$ and $r^{-}=p^{+} /\left(p^{+}-1\right)$.

Proof. Let

$$
r_{1}=\|u\|_{p(k)}, \quad r_{2}=\|v\|_{r(k)}
$$


then

$$
\begin{aligned}
\sum_{k \in Z} \frac{u(k) v(k)}{r_{1} r_{2}} & \leq \sum_{k \in Z}\left[\frac{1}{p(k)}\left(\frac{u(k)}{r_{1}}\right)^{p(k)}+\frac{1}{r(k)}\left(\frac{v(k)}{r_{2}}\right)^{r(k)}\right] \\
& \leq \frac{1}{p^{-}}+\frac{1}{r^{-}} .
\end{aligned}
$$

The proof is completed.

Lemma 12. Assume that all the conditions of Theorem 1 hold. Then the functional I satisfies the P.S. condition.

Proof. Assume that $\left\{u_{j}\right\}_{j \in N} \subset E$ is a sequence such that $\left\{I\left(u_{j}\right)\right\}$ is a bounded and $I^{\prime}\left(u_{j}\right) \rightarrow 0$ as $j \rightarrow+\infty$. Then there exists a positive constant $M^{\prime}$ such that $\left|I\left(u_{j}\right)\right| \leq M^{\prime}$ for all $j \in Z^{+}$.

First, we show that $\left\|u_{j}\right\|$ is bounded. Now we may assume that $\left\|u_{j}\right\|>1$; otherwise, $\left\|u_{j}\right\|$ is bounded obviously. When $j$ is large enough, we have

$$
\begin{aligned}
M^{\prime}+\frac{1}{\beta}\left\|u_{j}\right\| \geq & I\left(u_{j}\right)-\frac{1}{\beta}\left\langle I^{\prime}\left(u_{j}\right), u_{j}\right\rangle \\
= & \sum_{k \in Z}\left(\frac{1}{p(k)}-\frac{1}{\beta}\right)\left[a(k)\left|\Delta u_{j}(k-1)\right|^{p(k)}\right. \\
& \left.+q(k)\left|u_{j}(k)\right|^{p(k)}\right] \\
& +\sum_{k \in Z}\left[\frac{1}{\beta} f\left(k, u_{j}\right) u_{j}(k)-F\left(k, u_{j}(k)\right)\right] \\
\geq & \left(\frac{1}{p^{+}}-\frac{1}{\beta}\right)\left\|u_{j}\right\|^{p^{-}} .
\end{aligned}
$$

It follows from $\beta>p^{+}$and $p^{-}>1$ that there exists a constant $M_{*}>0$ such that

$$
\left\|u_{j}\right\| \leq M_{*}, \quad \forall j \in Z^{+} .
$$

By Lemma 10, we can choose a subsequence, still denoted by $\left\{u_{j}\right\}$, such that

$$
\begin{gathered}
u_{j} \rightarrow u_{*} \quad \text { in } E, \\
u_{j} \longrightarrow u_{*} \quad \text { in } l^{p(k)},
\end{gathered}
$$

for some $u_{*} \in E$.

Next, we prove that

$$
\begin{gathered}
\lim _{j \rightarrow+\infty} \sum_{k \in Z}\left(f\left(k, u_{j}(k)\right)-f\left(k, u_{*}(k)\right)\right) \\
\times\left(u_{j}(k)-u_{*}(k)\right)=0 .
\end{gathered}
$$

By (f), for any $0<\epsilon<\min \left\{1 / 2,1 / 2 p^{+}\right\}$, there exists a positive constant $\rho<1$ with $q_{*}^{\left(1 / p^{-}\right)} \rho^{\left(p^{+} / p^{-}\right)}<1$ such that

$$
f(k, u) \leq \epsilon q(k)|u|^{p(k)-1} \quad \forall k \in Z,|u| \leq \rho,
$$

Since $u_{*} \in E$, there exists a positive integer $T$ such that

$$
\left|u_{*}(k)\right| \leq \frac{\rho}{2} \quad \forall k>T
$$

we have

$$
\left|f\left(k, u_{*}(k)\right)\right| \leq \epsilon q(k)\left|u_{*}(k)\right|^{p(x)-1} \quad \forall k>T .
$$

By (30), there exists $\nu_{1} \in Z^{+}$such that

$$
\left|u_{j}(k)-u_{*}(k)\right| \leq \frac{\rho}{2} \quad \forall j>v_{1}, k \in Z .
$$

This, combined with (33) and (32), gives us

$$
\begin{gathered}
\left|u_{j}(k)\right| \leq\left|u_{j}(k)-u_{*}(k)\right|+\left|u_{*}(k)\right| \leq \rho \quad \forall j>v_{1}, k>T, \\
\left|f\left(k, u_{j}(k)\right)\right| \leq \epsilon q(k)\left|u_{j}(k)\right|^{p(k)-1} \quad \forall j>v_{1}, k>T .
\end{gathered}
$$

Then for $j>v_{1}$,

$$
\begin{aligned}
& \sum_{|k|>T}\left|f\left(k, u_{j}(k)\right)-f\left(k, u_{*}(k)\right)\right|\left|u_{j}(k)-u_{*}(k)\right| \\
& \leq \epsilon \sum_{|k|>T}\left(q(k)\left|u_{j}(k)\right|^{p(k)-1}+q(k)\left|u_{*}(k)\right|^{p(k)-1}\right) \\
& \quad \times\left(\left|u_{j}(k)\right|+\left|u_{*}(k)\right|\right) \\
& \leq \epsilon \sum_{k \in Z} q(k)^{1 / r(k)}\left|u_{j}(k)\right|^{p(k)-1} q(k)^{1 / p(k)}\left|u_{j}(k)\right| \\
& +\epsilon \sum_{k \in Z} q(k)^{1 / r(k)}\left|u_{j}(k)\right|^{p(k)-1} q(k)^{1 / p(k)}\left|u_{*}(k)\right| \\
& +\epsilon \sum_{k \in Z} q(k)^{1 / r(k)}\left|u_{*}(k)\right|^{p(k)-1} q(k)^{1 / p(k)}\left|u_{j}(k)\right| \\
& +\epsilon \sum_{k \in Z} q(k)^{1 / r(k)}\left|u_{*}(k)\right|^{p(k)-1} q(k)^{1 / p(k)}\left|u_{*}(k)\right|
\end{aligned}
$$

where $1 / r(k)+1 / p(k)=1$ for all $k \in Z$.

Let $v_{1}=\left\{v_{1}(k)\right\}$ and $v_{1}(k)=q(k)^{1 / r(k)}\left|u_{j}(k)\right|^{p(k)-1}$, $v_{2}=\left\{v_{2}(k)\right\}$ and $v_{2}(k)=q(k)^{1 / p(k)}\left|u_{j}(k)\right|, h_{1}=\left\{h_{1}(k)\right\}$ and $h_{1}(k)=q(k)^{1 / r(k)}\left|u_{*}(k)\right|^{p(k)-1}$, and $h_{2}=\left\{h_{2}(k)\right\}$ and $h_{2}(k)=q(k)^{1 / p(k)}\left|u_{*}(k)\right|$.

It is easy to check that $v_{1}, h_{1} \in l^{r(k)}$ and $v_{2}, h_{2} \in l^{p(k)}$. Then using Lemma 11, for $j>v_{1}$, we have

$$
\begin{gathered}
\sum_{|k|>T}\left|f\left(k, u_{j}(k)\right)-f\left(k, u_{*}(k)\right)\right|\left|u_{j}(k)-u_{*}(k)\right| \\
\leq\left(\frac{\epsilon}{p^{-}}+\frac{\epsilon}{r^{-}}\right) \\
\quad \times\left(\left\|v_{1}\right\|_{r(k)}\left\|v_{2}\right\|_{p(k)}+\left\|v_{1}\right\|_{r(k)}\left\|h_{2}\right\|_{p(k)}\right. \\
\left.\quad+\left\|h_{1}\right\|_{r(k)}\left\|v_{2}\right\|_{p(k)}+\left\|h_{1}\right\|_{r(k)}\left\|h_{2}\right\|_{p(k)}\right) .
\end{gathered}
$$


Now we show that $\left\|v_{1}\right\|_{r(k)}$ is bounded. We may assume that $\left\|v_{1}\right\|_{r(k)}>1$; otherwise, $\left\|v_{1}\right\|_{r(k)}$ is bounded obviously. By Lemmas 4 and 5 , we have

$$
\left\|v_{1}\right\|_{r(k)} \leq\left[\sum_{k \in Z} q(k)\left|u_{j}(k)\right|^{p^{(k)}}\right]^{1 / r^{-}} \leq\left\|u_{j}\right\|^{p^{+} / r^{-}} \leq M_{*}^{p^{+} / r^{-}} .
$$

Let $M_{1}=\left\{1, M_{*}^{p^{+} / r^{-}}\right\}$; then $\left\|v_{1}\right\|_{r(k)} \leq M_{1}$; that is, $\left\|v_{1}\right\|_{r(k)}$ is bounded. Using the similar arguments as above, we obtain that $\left\|v_{2}\right\|_{p(k)},\left\|h_{1}\right\|_{r(k)}$, and $\left\|h_{2}\right\|_{p(k)}$ are bounded; that is, there exist three positive constants $M_{2}, M_{3}$, and $M_{4}$ such that

$$
\left\|v_{2}\right\|_{p(k)} \leq M_{2}, \quad\left\|h_{1}\right\|_{r(k)} \leq M_{3}, \quad\left\|h_{2}\right\|_{p(k)} \leq M_{4} .
$$

This, combined with (38), gives us

$$
\begin{aligned}
\sum_{|k|>T}\left|f\left(k, u_{j}(k)\right)-f\left(k, u_{*}(k)\right)\right|\left|u_{j}(k)-u_{*}(k)\right| \\
\leq \epsilon\left(\frac{1}{p^{-}}+\frac{1}{r^{-}}\right) \\
\quad \times\left(M_{1} M_{2}+M_{1} M_{4}+M_{3} M_{2}+M_{3} M_{4}\right) \quad \forall j>v_{1} .
\end{aligned}
$$

By continuity of the finite sum and (30), there exists $v_{2} \in Z^{+}$ such that

$$
\begin{aligned}
& \sum_{|k| \leq T}\left|f\left(k, u_{j}(k)\right)-f\left(k, u_{*}(k)\right)\right| \\
& \quad \times\left|u_{j}(k)-u_{*}(k)\right| \leq \epsilon \quad \forall j \geq v_{2} .
\end{aligned}
$$

Let $v=\max \left\{v_{1}, v_{2}\right\}$. Combining (41) and (42) together, we have

$$
\begin{aligned}
\sum_{k \in Z}\left|f\left(k, u_{j}(k)\right)-f\left(k, u_{*}(k)\right)\right|\left|u_{j}(k)-u_{*}(k)\right| \\
\leq \epsilon\left(\frac{1}{p^{-}}+\frac{1}{r^{-}}\right) \\
\quad \times\left(M_{1} M_{2}+M_{1} M_{4}+M_{3} M_{2}+M_{3} M_{4}\right)+\epsilon \quad \forall j>v .
\end{aligned}
$$

Thus, (31) holds.

Finally, we show that $\left\{u_{j}\right\}$ possesses a convergent subsequence. Since $I^{\prime}\left(u_{j}\right) \rightarrow 0$ and $u_{j} \rightarrow u_{*}$, it follows at once that

$$
\lim _{j \rightarrow+\infty}\left\langle I^{\prime}\left(u_{j}\right)-I^{\prime}\left(u_{*}\right), u_{j}-u_{*}\right\rangle=0 .
$$

This, combined with (31), gives us

$$
\begin{gathered}
\lim _{j \rightarrow+\infty}\left\{\sum _ { k \in Z } a ( k ) \left[\left|\Delta u_{j}(k-1)\right|^{p(k)-2} \Delta u_{j}(k-1)\right.\right. \\
\left.-\left|\Delta u_{*}(k-1)\right|^{p(k)-2} \Delta u_{*}(k-1)\right] \\
\times\left(\Delta u_{j}(k-1)-\Delta u_{*}(k-1)\right) \\
+\sum_{k \in Z} q(k)\left[\left|u_{j}(k)\right|^{p(k)-2} u_{j}(k)\right. \\
\left.-\left|u_{*}(k)\right|^{p(k)-2} u_{*}(k)\right] \\
\left.\times\left(u_{j}(k)-u_{*}(k)\right)\right\}=0 .
\end{gathered}
$$

The following two inequalities are taken from [20] and will play an important role in the proof of our main result:

$$
\begin{gathered}
{\left[\left(|\xi|^{p-2} \xi-|\eta|^{p-2} \eta\right)(\xi-\eta)\right]} \\
\times(|\xi|+|\eta|)^{2-p} \geq|\xi-\eta|^{2}, \quad 1<p<2, \\
2^{p}\left[\left(|\xi|^{p-2} \xi-|\eta|^{p-2} \eta\right)(\xi-\eta)\right] \geq|\xi-\eta|^{p}, \quad p \geq 2,
\end{gathered}
$$

for every $\xi$ and $\eta$ in $R$. We define

$$
\begin{aligned}
R_{j}(k)= & a(k)\left[\left|\Delta u_{j}(k-1)\right|^{p(k)-2} \Delta u_{j}(k-1)\right. \\
& \left.-\left|\Delta u_{*}(k-1)\right|^{p(k)-2} \Delta u_{*}(k-1)\right] \\
& \times\left(\Delta u_{j}(k-1)-\Delta u_{*}(k-1)\right) \quad \forall k \in Z . \\
Q_{j}(k)= & q(k)\left[\left|u_{j}(k)\right|^{p(k)-2} u_{j}(k)-\left|u_{*}(k)\right|^{p(k)-2} u_{*}(k)\right] \\
& \times\left(u_{j}(k)-u_{*}(k)\right) \quad \forall k \in Z .
\end{aligned}
$$

This, combined with (45), produces at once

$$
\lim _{j \rightarrow+\infty} \sum_{k \in Z} R_{j}(k)=0, \quad \lim _{j \rightarrow+\infty} \sum_{k \in Z} Q_{j}(k)=0 .
$$

Now we show that $\varphi\left(u_{j}-u_{*}\right) \rightarrow 0$ as $j \rightarrow+\infty$. That is,

$$
\begin{gathered}
\lim _{j \rightarrow+\infty} \sum_{k \in Z} a(k)\left|\Delta u_{j}(k-1)-\Delta u_{*}(k-1)\right|^{p(k)}=0, \\
\lim _{j \rightarrow+\infty} \sum_{k \in Z} q(k)\left|u_{j}(k)-u_{*}(k)\right|^{p(k)}=0 .
\end{gathered}
$$

Let us first prove (50). Since $\left\{u_{j}\right\}$ and $u_{*}$ are bounded in $E$, there exists a constant $M^{*}>1$ such that $\varphi\left(u_{j}\right) \leq M^{*}$ and $\varphi\left(u_{*}\right) \leq M^{*}$ for all $j \in Z^{+}$. We denote

$$
\begin{aligned}
& W_{1}=\{k \in Z: 1<p(k)<2\}, \\
& W_{2}=\{k \in Z: p(k) \geq 2\} .
\end{aligned}
$$


By (46), we have

$$
\begin{aligned}
& \sum_{k \in W_{2}} q(k)\left|u_{j}(k)-u_{*}(k)\right|^{p(k)} \\
& \leq \sum_{k \in W_{2}} 2^{p^{+}} q(k)\left(\left|u_{j}(k)\right|^{p(k)-2} u_{j}(k)\right. \\
& \left.-\left|u_{*}(k)\right|^{p(k)-2} u_{*}(k)\right) \\
& \times\left(u_{j}(k)-u_{*}(k)\right) \leq 2^{p^{+}} \sum_{k \in Z} Q_{j}(k), \\
& \sum_{k \in W_{1}} q(k)\left|u_{j}(k)-u_{*}(k)\right|^{p(k)} \\
& =\sum_{k \in W_{1}} q(k)\left[\left|u_{j}(k)-u_{*}(k)\right|^{2}\right]^{p(k) / 2} \\
& \leq \sum_{k \in W_{1}}\left(Q_{j}(k)\right)^{p(k) / 2} \\
& \times\left\{q(k)\left[\left|u_{j}(k)\right|+\left|u_{*}(k)\right|\right]^{p(k)}\right\}^{(2-p(k)) / 2} \\
& \leq \sum_{k \in W_{1}}\left(Q_{j}(k)\right)^{p(k) / 2} \\
& \times\left[2^{p^{+}} q(k)\left(\left|u_{j}(k)\right|^{p^{(k)}}+\left|u_{*}(k)\right|^{p(k)}\right)\right]^{(2-p(k)) / 2} \\
& \leq 2^{p^{+}\left(2-p^{-}\right) / 2} \\
& \times \sum_{k \in Z}\left(Q_{j}(k)\right)^{p(k) / 2} \\
& \times\left[q(k)\left|u_{j}(k)\right|^{p(k)}+q(k)\left|u_{*}(k)\right|^{p(k)}\right]^{(2-p(k)) / 2} .
\end{aligned}
$$

Let

$$
\begin{aligned}
p_{1}(k) & =\frac{2}{p(k)}, \quad r_{1}(k)=\frac{2}{2-p(k)}, \\
v_{j *}(k) & =\left(Q_{j}(k)\right)^{p(k) / 2}, \\
w_{j *}(k) & =\left[q(k)\left|u_{j}(k)\right|^{p(k)}+q(k)\left|u_{*}(k)\right|^{p(k)}\right]^{(2-p(k)) / 2} .
\end{aligned}
$$

Then it is easy to check that $v_{j *}=\left\{v_{j *}(k)\right\} \in l_{p_{1}(k)}$ and $w_{j *}=$ $\left\{w_{j *}(k)\right\} \in l_{r_{1}(k)}$. By Lemma 11 and (53), we have

$$
\begin{aligned}
& \sum_{k \in W_{1}} q(k)\left|u_{j}(k)-u_{*}(k)\right|^{p(k)} \\
& \quad \leq 2^{p^{+}\left(2-p^{-}\right) / 2}\left(1+\frac{p^{+}}{2}-\frac{p^{-}}{2}\right)\left\|v_{j *}\right\|_{p_{1}(k)}\left\|w_{j *}\right\|_{r_{1}(k)}
\end{aligned}
$$

Since

$$
\begin{gathered}
\lim _{j \rightarrow+\infty} \sum_{k \in Z} Q_{j}(k)=0, \\
\sum_{k \in Z}\left[q(k)\left|u_{j}(k)\right|^{p(k)}+q(k)\left|u_{*}(k)\right|^{p(k)}\right] \\
\leq \varphi\left(u_{j}\right)+\varphi\left(u_{*}\right) \leq 2 M^{*} .
\end{gathered}
$$

It is easy to see that $\left\|v_{j *}\right\|_{p_{1}(k)} \rightarrow 0$ as $j \rightarrow+\infty$ and $\left\|w_{j *}\right\|_{r_{1}(k)}$ is bounded for all $j \in Z^{+}$. This, combined with (52) and (55), gives us

$$
\begin{aligned}
\lim _{j \rightarrow+\infty} \sum_{k \in Z} q(k)\left|u_{j}(k)-u_{*}(k)\right|^{p(k)} & \\
= & \lim _{j \rightarrow+\infty} \sum_{k \in W_{1}} q(k)\left|u_{j}(k)-u_{*}(k)\right|^{p(k)} \\
& +\lim _{j \rightarrow+\infty} \sum_{k \in W_{2}} q(k)\left|u_{j}(k)-u_{*}(k)\right|^{p(k)}=0 .
\end{aligned}
$$

Using the similar arguments, we have (49). So $\varphi\left(u_{j}-u_{*}\right) \rightarrow 0$ as $j \rightarrow+\infty$. By Lemma 5, it follows that $\left\|u_{j}-u_{*}\right\| \rightarrow 0$ as $j \rightarrow+\infty$, and the proof is completed.

Proof of Theorem 1. The proof consists of two steps.

Step 1. We use Lemma 9 to show that (1) has a nontrivial solution in $E$.

First we prove that $I$ satisfies $\left(J_{1}\right)$ of Lemma 9. It follows from (32) that

$$
|F(k, u(k))| \leq \frac{1}{2 p^{+}} q(k)|u(k)|^{p(k)}
$$

for $|u(k)| \leq \rho$ and $k \in Z$. Then, let $\delta=q_{*}^{\left(1 / p^{-}\right)} \rho^{\left(p^{+} / p^{-}\right)}<1$, for all $u \in \partial B_{\delta} \cap E$, we have $|u(k)| \leq \rho$ and

$$
\begin{aligned}
I(u)= & \sum_{k \in Z}\left[\frac{a(k)}{p(k)}|\Delta u(k-1)|^{p(k)}+\frac{q(k)}{p(k)}|u(k)|^{p(k)}\right] \\
& -\sum_{k \in Z} F(k, u(k)) \\
\geq & \sum_{k \in Z} \frac{1}{p^{+}}\left[a(k)|\Delta u(k-1)|^{p(k)}+q(k)|u(k)|^{p(k)}\right] \\
& -\sum_{k \in Z} \frac{1}{2 p^{+}} q(k)|u(k)|^{p(k)} \\
\geq & \sum_{k \in Z} \frac{1}{2 p^{+}}\left[a(k)|\Delta u(k-1)|^{p(k)}+q(k)|u(k)|^{p(k)}\right] \\
\geq & \frac{1}{2 p^{+}}\|u\|^{p^{+}}=\frac{1}{2 p^{+}} \delta^{p^{+}}>0,
\end{aligned}
$$

and hence $I$ satisfies $\left(J_{1}\right)$ of Lemma 9. 
Next, we prove that $I$ satisfies $\left(\mathrm{J}_{2}\right)$ of Lemma 9. Let $e=$ $\{e(k)\} \in E$ and

$$
e(k)= \begin{cases}0, & \text { if } k \neq 0 \\ 1, & \text { if } k=0\end{cases}
$$

Then

$$
F(k, e(k))= \begin{cases}0, & \text { if } k \neq 0 \\ F(0,1), & \text { if } k=0\end{cases}
$$

By Lemma 7, for $s>1$, we have

$$
\begin{array}{ll}
\sum_{k \in Z} F_{1}(k, s e(k))=F_{1}(0, s) \geq s^{\beta} F_{1}(0,1), & F_{1}(0,1)>0, \\
\sum_{k \in Z} F_{2}(k, s e(k))=F_{2}(0, s) \leq s^{\tau} F_{2}(0,1), & F_{2}(0,1) \geq 0 .
\end{array}
$$

Then

$$
\begin{aligned}
I(s e)= & \sum_{k \in Z}\left[\frac{a(k)}{p(k)}|s \Delta e(k-1)|^{p(k)}+\frac{q(k)}{p(k)}|s e(k)|^{p(k)}\right] \\
& -\sum_{k \in Z}\left[F_{1}(k, s e(k))-F_{2}(k, s e(k))\right] \\
\leq & \frac{a(0)}{p(0)} s^{p(0)}+\frac{a(1)}{p(1)} s^{p(1)}+\frac{q(0)}{p(0)} s^{p(0)} \\
& -s^{\beta} F_{1}(0,1)+s^{\tau} F_{2}(0,1) .
\end{aligned}
$$

Since $F_{1}(0,1)>0$ and $p(0), p(1), \tau$ are smaller than $\beta$, we can choose $s^{*}$ large enough such that $I\left(s^{*} e\right)<0$. So we have verified all assumptions of Lemma 9; we know that $I$ possesses a critical value $\alpha \geq\left(1 / 2 p^{+}\right) \delta^{p^{+}}>0$, where

$$
\begin{aligned}
& \alpha=\inf _{h \in \Gamma} \max _{s \in[0,1]} I(h(s)), \\
& \Gamma=\left\{h \in C([0,1], E): h(0)=0, h(1)=s^{*} e\right\} .
\end{aligned}
$$

A critical point $u^{*}$ of $I$ corresponding to $\alpha$ is nonzero as $\alpha>0$.

Step 2. We prove that (1) has a ground state in $E$.

Let

$$
K=\left\{u \in E: I^{\prime}(u)=0, u \neq 0\right\}
$$

be the critical set of $I$. Obviously, $K$ is a nonempty set. Denote

$$
\widetilde{c}=\inf \{I(u): u \in K\} .
$$

Since $u \in K$, we have

$$
I(u)=I(u)-\frac{1}{\beta}\left\langle I^{\prime}(u), u\right\rangle \geq\left(\frac{1}{p^{+}}-\frac{1}{\beta}\right) \varphi(u) \geq 0 .
$$

Then $0 \leq \tilde{c} \leq I(u)$.
Suppose that $\left\{u_{j}\right\} \subset K$ such that $I\left(u_{j}\right) \rightarrow \widetilde{c}$. Obviously, $\left\{u_{j}\right\}$ is a P.S. sequence. By Lemma 12, we can choose a subsequence, still denoted by $\left\{u_{j}\right\}$, such that

$$
\lim _{j \rightarrow \infty}\left\|u_{j}-u_{0}\right\|=0, \quad u_{0} \in E .
$$

Then $I\left(u_{j}\right) \rightarrow I\left(u_{0}\right)$ and $I\left(u_{0}\right)=\widetilde{c}$. Now we prove that $u_{0}$ is nonzero. If $u_{0}=0$, then there exists a positive integer $W$ such that for all $j>W$, we have

$$
\left\|u_{j}\right\| \leq q_{*}^{\left(1 / p^{-}\right)} \rho^{\left(p^{+} / p^{-}\right)}<1 .
$$

By (32), it follows that

$$
\begin{aligned}
\left\langle I^{\prime}\left(u_{j}\right), u_{j}\right\rangle= & \sum_{k \in Z}\left[a(k)\left|\Delta u_{j}(k-1)\right|^{p(k)}+q(k)\left|u_{j}(k)\right|^{p(k)}\right. \\
& \left.\quad-f\left(k, u_{j}(k)\right) u_{j}(k)\right] \\
\geq & \sum_{k \in Z}\left[a(k)\left|\Delta u_{j}(k-1)\right|^{p(k)}\right. \\
& \left.+q(k)\left|u_{j}(k)\right|^{p(k)}\right] \\
& -\frac{1}{2} \sum_{k \in Z} q(k)\left|u_{j}(k)\right|^{p(k)} \\
\geq & \frac{1}{2} \varphi\left(u_{j}\right) \geq \frac{1}{2}\left\|u_{j}\right\|^{p^{+}}>0,
\end{aligned}
$$

which is in contradiction to $I^{\prime}\left(u_{j}\right)=0$. Thus, $u_{0}$ is the ground state solution of (1). The proof is completed.

\section{Conflict of Interests}

The author declares that there is no conflict of interests regarding the publication of this paper.

\section{Acknowledgments}

This work was partially supported by the Foundation for Distinguished Young Talents in Higher Education of Guangdong, China (no. 2013LYM-0093.), by Research Fund for the Doctoral Program of Higher Education of China (no. 20124410110001), and by Science Foundation for Young Teachers of Wuyi University (no. 2013zk02.).

\section{References}

[1] T. Bartsch and Z. Liu, "On a superlinear elliptic $p$-Laplacian equation," Journal of Differential Equations, vol. 198, no. 1, pp. 149-175, 2004.

[2] S. Liu, "On ground states of superlinear $p$-Laplacian equations in $\mathbf{R}^{N}$," Journal of Mathematical Analysis and Applications, vol. 361, no. 1, pp. 48-58, 2010.

[3] J. S. W. Wong, "On the generalized Emden-Fowler equation," SIAM Review, vol. 17, pp. 339-360, 1975. 
[4] X. Fan and X. Han, "Existence and multiplicity of solutions for $p(x)$-Laplacian equations in $R^{N}$," Nonlinear Analysis. Theory, Methods \& Applications. An International Multidisciplinary Journal A: Theory and Methods, vol. 59, no. 1-2, pp. 173-188, 2004.

[5] X.-L. Fan and Q.-H. Zhang, "Existence of solutions for $p(x)$ Laplacian Dirichlet problem," Nonlinear Analysis. Theory, Methods \& Applications. An International Multidisciplinary Journal A: Theory and Methods, vol. 52, no. 8, pp. 1843-1852, 2003.

[6] X. Fan and D. Zhao, "On the spaces $L^{p(x)}(\Omega)$ and $W^{m, p(x)}(\Omega)$," Journal of Mathematical Analysis and Applications, vol. 263, no. 2, pp. 424-446, 2001.

[7] X. Fan, Y. Zhao, and D. Zhao, "Compact imbedding theorems with symmetry of Strauss-Lions type for the space $W^{1, p(x)}(\Omega)$," Journal of Mathematical Analysis and Applications, vol. 255, no. 1, pp. 333-348, 2001.

[8] R. P. Agarwal, Difference Equations and Inequalities, Monographs and Textbooks in Pure and Applied Mathematics, Marcel Dekker, New York, NY, USA, 2nd edition, 2000, Theory, methods, and applications.

[9] R. P. Agarwal, K. Perera, and D. O’Regan, "Multiple positive solutions of singular and nonsingular discrete problems via variational methods," Nonlinear Analysis. Theory, Methods \& Applications. An International Multidisciplinary Journal A: Theory and Methods, vol. 58, no. 1-2, pp. 69-73, 2004.

[10] F. M. Atici and G. Sh. Guseinov, "Positive periodic solutions for nonlinear difference equations with periodic coefficients," Journal of Mathematical Analysis and Applications, vol. 232, no. 1, pp. 166-182, 1999.

[11] Z. Guo and J. Yu, "Existence of periodic and subharmonic solutions for second-order superlinear difference equations," Science in China A. Mathematics, vol. 46, no. 4, pp. 506-515, 2003.

[12] Z. Guo and J. Yu, "The existence of periodic and subharmonic solutions of subquadratic second order difference equations," Journal of the London Mathematical Society, vol. 68, no. 2, pp. 419-430, 2003.

[13] M. Ma and Z. Guo, "Homoclinic orbits and subharmonics for nonlinear second order difference equations," Nonlinear Analysis. Theory, Methods \& Applications. An International Multidisciplinary Journal A: Theory and Methods, vol. 67, no. 6, pp. 1737-1745, 2007.

[14] M. Ma and Z. Guo, "Homoclinic orbits for second order selfadjoint difference equations," Journal of Mathematical Analysis and Applications, vol. 323, no. 1, pp. 513-521, 2006.

[15] J. Yu, Z. Guo, and X. Zou, "Periodic solutions of second order self-adjoint difference equations," Journal of the London Mathematical Society, vol. 71, no. 1, pp. 146-160, 2005.

[16] A. Iannizzotto and S. A. Tersian, "Multiple homoclinic solutions for the discrete $p$-Laplacian via critical point theory," Journal of Mathematical Analysis and Applications, vol. 403, no. 1, pp. 173182, 2013.

[17] P. Chen, X. H. Tang, and R. P. Agarwal, "Existence of homoclinic solutions for $p(n)$-Laplacian Hamiltonian systems on Orlicz sequence spaces," Mathematical and Computer Modelling, vol. 55, no. 3-4, pp. 989-1002, 2012.

[18] J. Lindenstrauss and L. Tzafriri, "On Orlicz sequence spaces," Israel Journal of Mathematics, vol. 10, pp. 379-390, 1971.

[19] K. Lindberg, "On subspaces of Orlicz sequence spaces," Polska Akademia Nauk. Instytut Matematyczny. Studia Mathematica, vol. 45, pp. 119-146, 1973.
[20] A. El Hamidi, "Existence results to elliptic systems with nonstandard growth conditions," Journal of Mathematical Analysis and Applications, vol. 300, no. 1, pp. 30-42, 2004. 


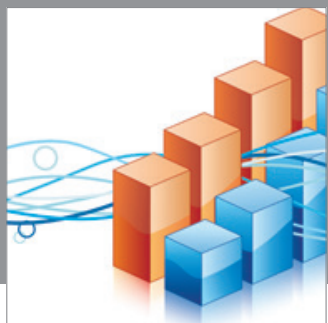

Advances in

Operations Research

mansans

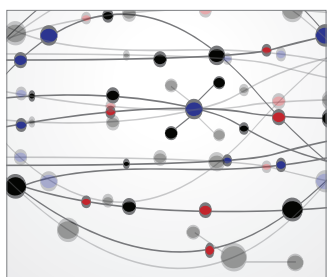

The Scientific World Journal
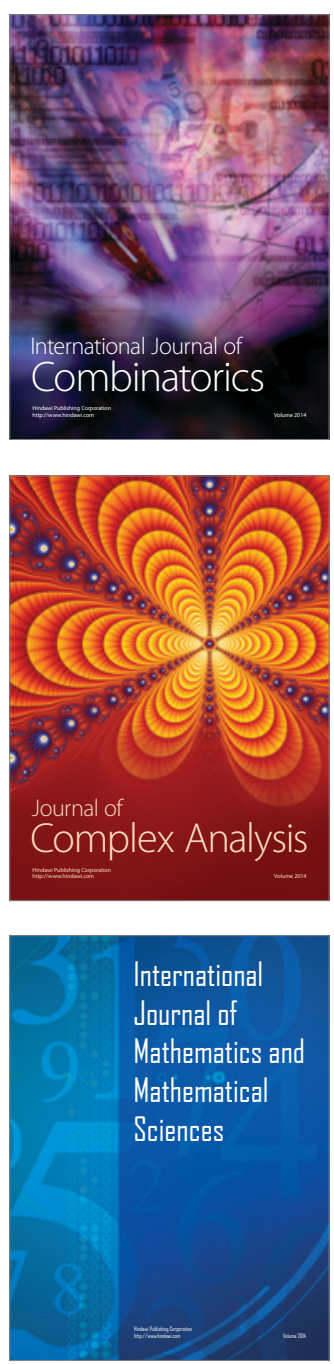
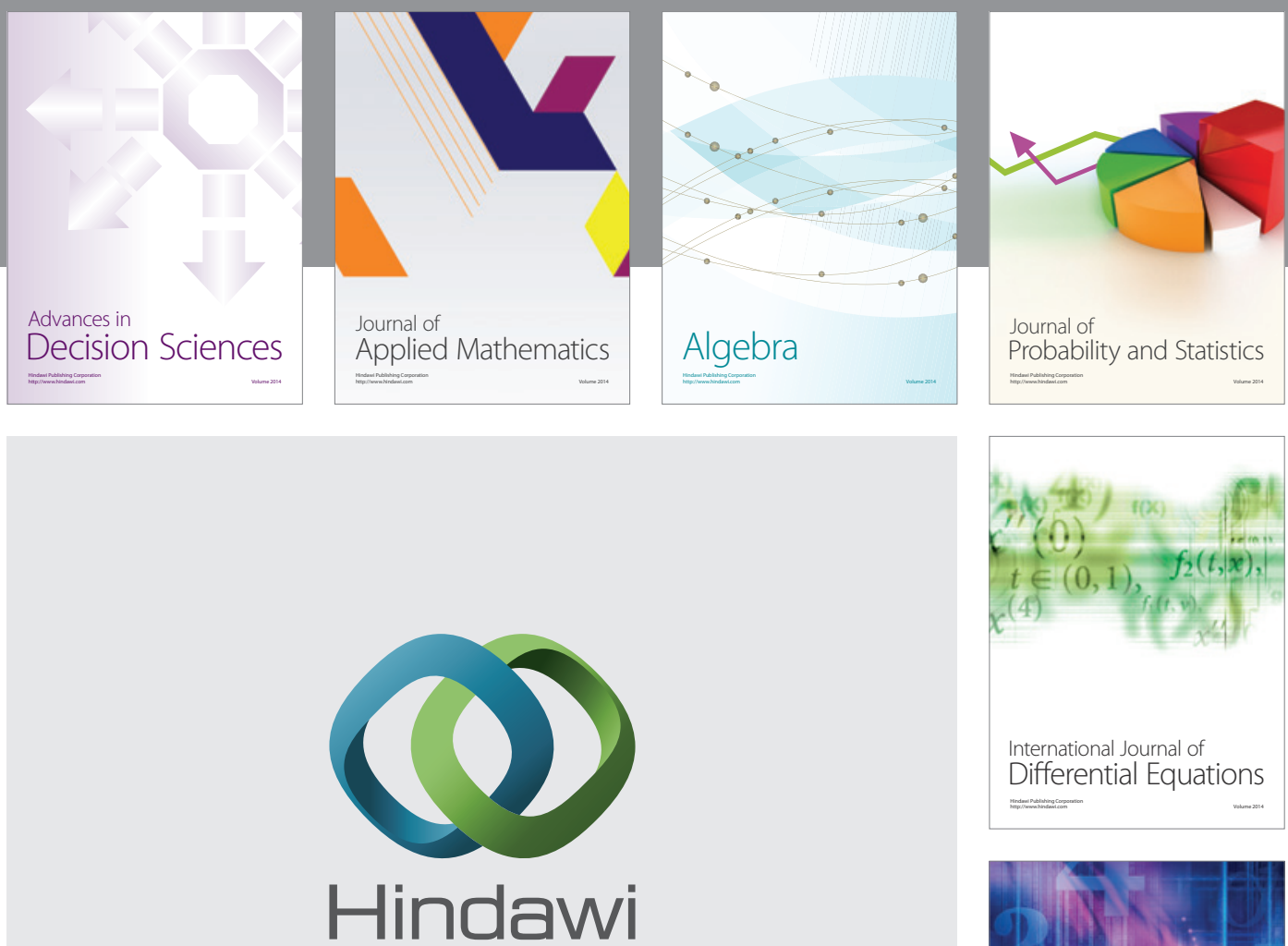

Submit your manuscripts at http://www.hindawi.com
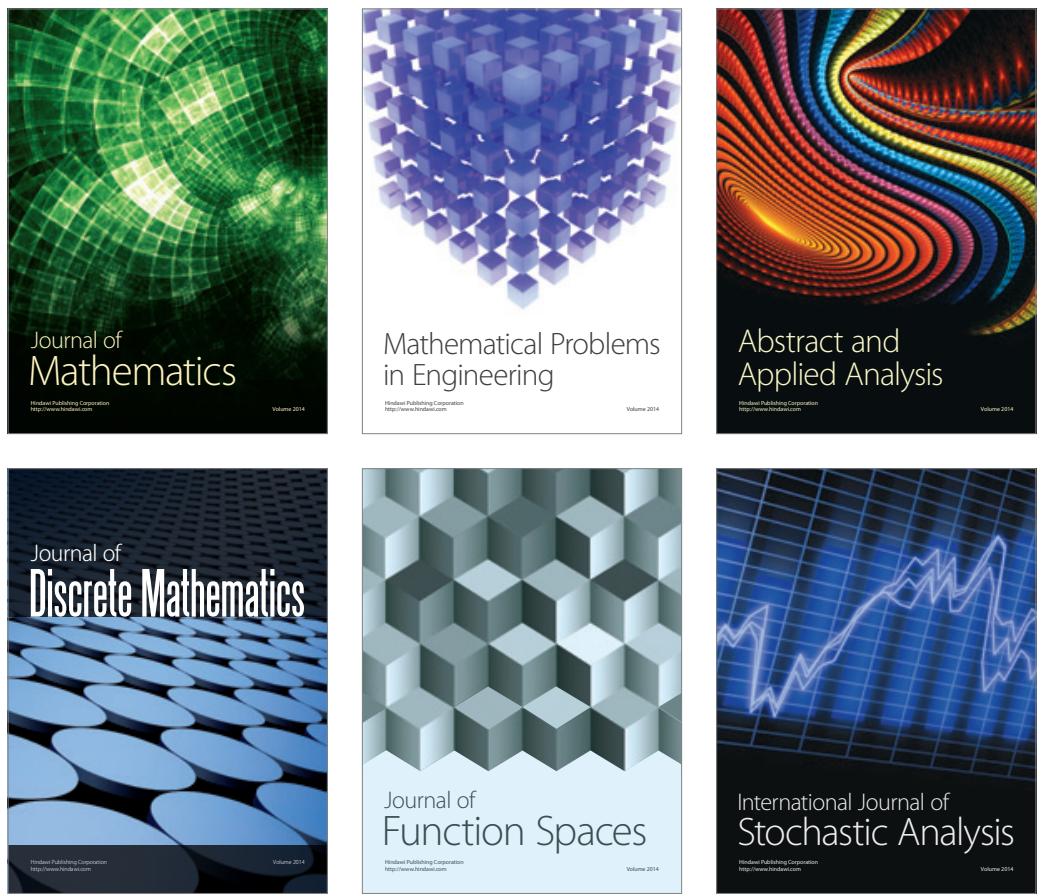

Journal of

Function Spaces

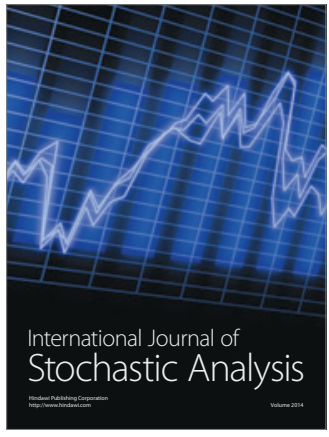

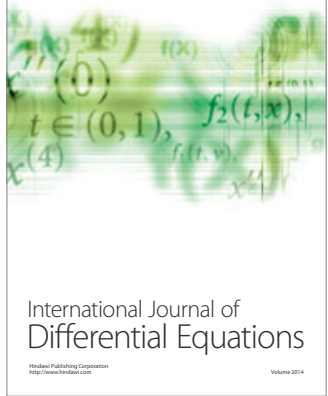
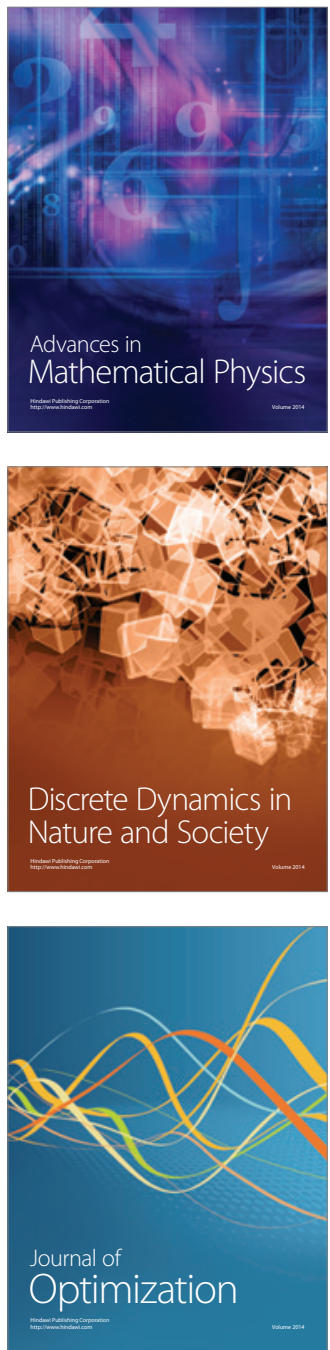